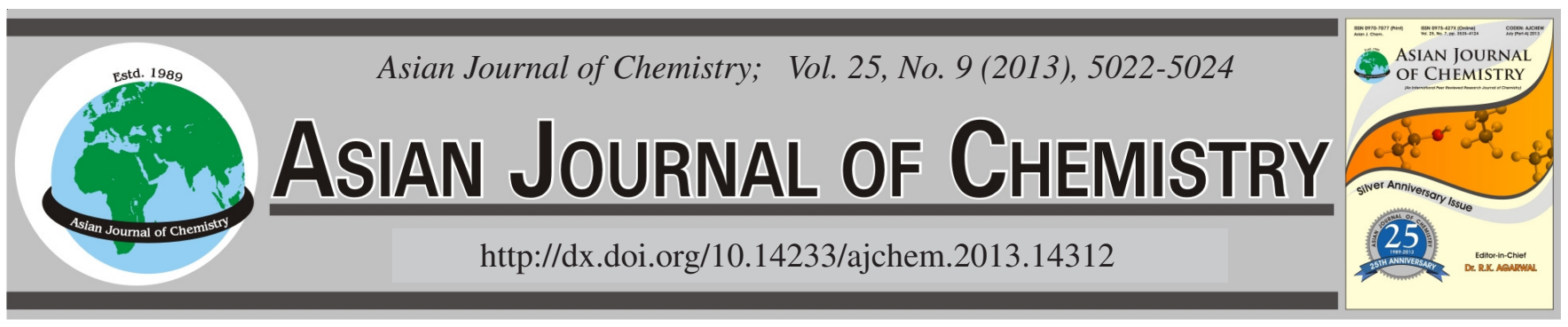

\title{
Synthesis and Crystal Structure of 5,5'-Dimethoxy-2,2'-[(hexane-1,6-diyldioxy)bis(nitrilomethylidyne)]diphenol
}

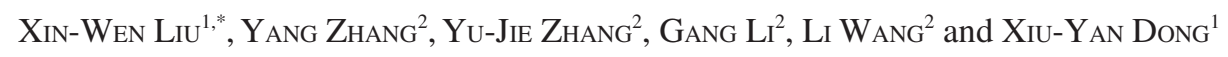

${ }^{1}$ School of Biological Science and Chemistry, Tianshui Normal University, Tianshui 741001, P.R. China

${ }^{2}$ School of Chemical and Biological Engineering, Lanzhou Jiaotong University, Lanzhou 730070, P.R. China

*Corresponding author: E-mail: liuxwts@126.com

(Received: 16 July 2012;

Accepted: 15 March 2013)

AJC-13127

\begin{abstract}
The chelating bis-oxime compound i.e., 5,5'-dimethoxy-2,2'-[(hexane-1,6-diyldioxy)bis(nitrilomethylidyne)]diphenol with the molecular formula $\mathrm{C}_{22} \mathrm{H}_{28} \mathrm{~N}_{2} \mathrm{O}_{6}$, assumes an $\mathrm{E}$ configuration with respect to the azomethine $\mathrm{C}=\mathrm{N}$ bond and the two phenyl rings are parallel to each other. There are two fairly strong intramolecular $\mathrm{O} 2-\mathrm{H} 2 \cdots \mathrm{N} 1$ and $\mathrm{O} 5-\mathrm{H} 5 \cdots \mathrm{N} 2$ hydrogen bonds. Each compound molecule links four other neighboring molecules through three pairs of intermolecular $\mathrm{C} 11-\mathrm{H} 11 \mathrm{C} \cdots \mathrm{O} 5$ and $\mathrm{C} 18-\mathrm{H} 18 \cdots \mathrm{O} 6$ hydrogen-bonding interactions into an infinite wave-like 2D-layer supramolecular structure.
\end{abstract}

Key Words: Salen-type bisoxime, Synthesis, Crystal structure.

ᄂ - - - - - - - - - - - - - - - - - - - - - - - - -

\section{INTRODUCTION}

Particular attention has been paid in recent years to the synthesis and crystal structure of dioxime compounds and their analogues due to their strong coordination capability ${ }^{1-3}$. Some of them or their metal complexes are used as biological models in understanding the structure of biomolecules and biological processes and they can also be used as invarious organic reaction processes as catalysts, models of reaction centers of metalloenzymes, fascinating magnetic properties and nonlinear optical materials ${ }^{4,5}$. Recently, a new series of salen-type bisoxime compounds have been synthesized by using an O-alkyloxime (-CH=N-O- $\left.\left(\mathrm{CH}_{2}\right)_{\mathrm{n}}-\mathrm{O}-\mathrm{N}=\mathrm{CH}-\right)$ unit $^{6}$, the large electronegativity of $\mathrm{O}$ atoms is expected to affect strongly the electronic properties of $\mathrm{N}_{2} \mathrm{O}_{2}$ coordination sphere ${ }^{7}$, which can lead to different and novel properties and structures of the resulted complexes ${ }^{8,9}$. Herein, we report on the synthesis and crystal structure of salen-type bisoxime compound, 5,5'dimethoxy-2,2'-[(hexane-1,6-diyldioxy)bis(nitrilomethylidyne)]diphenol.

\section{EXPERIMENTAL}

2-Hydroxy-4-methoxybenzaldehyde was purchased from Alfa Aesar and used without further purification. 1,6Bis(aminooxy)hexane was synthesized according to an analogous method reported earlier ${ }^{10}$. The other reagents and solvents were analytical grade reagents from Tianjing Chemical Reagent Factory. C, H and N analyses were carried out with a GmbH VariuoEL V3.00 automatic elemental analyzer. X-Ray single crystal structure was determined on a Bruker Smart 1000 CCD area detector. Melting points were measured by the use of a microscopic melting point apparatus made in Beijing Taike Instrument Limited Company and the thermometer was uncorrected.

General procedure: 5,5'-Dimethoxy-2,2'-[(hexane1,6diyldioxy)- $b i$ (nitrilomethylidyne)]diphenol was synthesized according to method reported earlier ${ }^{11}$. To an ethanolic solution (6 mL) of 2-hydroxy-4-methoxybenzaldehyde (194.5 mg, 1.28 mmol) was added an ethanol solution $(10 \mathrm{~mL})$ of $1,6-$ bis(aminooxy)hexane $(81.1 \mathrm{mg}, 0.64 \mathrm{mmol})$. The reaction mixture was stirred at 328-333 $\mathrm{K}$ for $6 \mathrm{~h}$. The formed precipitate was separated by filtration and washed successively with ethanol $/ n$-hexane (1:4). The product was dried under vacuum to yield $123.78 \mathrm{mg}$ of the title compound. Yield, $46.5 \%$. mp. 349-350 K. Anal. calcd. (\%) for $\mathrm{C}_{22} \mathrm{H}_{28} \mathrm{~N}_{2} \mathrm{O}_{6}$ : C, 63.45; H, 6.78; $\mathrm{N}, 6.73$. Found (\%): C, 63.96; H, 6.56; N, 6.55.

The title compound ( $5 \mathrm{mg}, 0.012 \mathrm{mmol}$ ) was dissolved in dichloromethane $(2 \mathrm{~mL})$ and ethanol $(2 \mathrm{~mL})$, the mixed solution was allowed to stand at room temperature for about one month. When the solution was partially evaporated, several colourless needle-like single crystals suitable for X-ray crystallographic analysis were obtained.

X-Ray structure determination: The single crystal of the title compound, with approximate dimensions of $0.43 \mathrm{~mm}$ $\times 0.18 \mathrm{~mm} \times 0.08 \mathrm{~mm}$ was placed on a Bruker Smart 1000 diffractmeter equipped with Apex CCD area detector. The 
diffraction data were collected using a graphite monochromated $\operatorname{MoK}_{\alpha}$ radition $(\lambda=0.71073 \AA)$ at $298(2) \mathrm{K}$. The structure was solved by using the program SHELXS-97 and Fourier difference techniques and refined by full-matrix least-squares method on $\mathrm{F}^{2}$ using SHELXL-97. Details of the data collection and refinements of the title compound are given in Table- 1 . The non-hydrogen atoms were refined anisotropically. Hydrogen atoms were added theoretically. CCDC: 890890.

\begin{tabular}{ll}
\multicolumn{2}{c}{ TABLE-1 } \\
\multicolumn{1}{c}{ CRYSTAL DATA AND STRUCTURE } \\
\multicolumn{1}{c}{$\mathrm{C}_{22} \mathrm{H}_{28} \mathrm{~N}_{2} \mathrm{O}_{6}$} \\
\hline Empirical formula & 416.46 \\
Formula weight & $298(2)$ \\
Temperature $(\mathrm{K})$ & 0.71073 \\
Wavelength $(\AA)$ & Triclinic \\
Crystal system & $\mathrm{P}-1$ \\
Space group & $\mathrm{a}=8.0680(10), \mathrm{b}=10.4611(12)$, \\
Cell dimensions $(\AA, \mathrm{A}, \mathrm{deg})$ & $\mathrm{c}=13.4759(16), \alpha=84.074(2)$, \\
& $\beta=73.9060(10), \gamma=83.976(2)$ \\
& $1083.5(2)$ \\
Volume $\left(\AA^{3}\right)$ & 2 \\
$\mathrm{Z}$ & 1.276 \\
Density (calculated) $\left(\mathrm{mg} / \mathrm{m}^{3}\right)$ & 0.093 \\
Absorption coefficient $\left(\mathrm{mm}{ }^{-1}\right)$ & 444.0 \\
$\mathrm{~F}_{(000)}$ & $-9 \leq \mathrm{h} \leq 9,-11 \leq \mathrm{k} \leq 12,-15 \leq 1$ \\
Index ranges & $\leq 16$ \\
& $5660 / 3747\left[\mathrm{R}_{\text {(int) }}=0.0681\right]$ \\
Reflections collected & 404 \\
Independent reflections & $3747 / 0 / 273$ \\
Data/restraints/parameters & 0.987 \\
Goodness of fit indicator & $\mathrm{R}_{1}=0.0562, \mathrm{wR}_{2}=0.0927$ \\
$\mathrm{R}[\mathrm{I}>2 \sigma(\mathrm{I})]$ & 0.195 and -0.190 \\
Largest diff. peak and hole & \\
$\left(\mathrm{e} \AA^{-3}\right)$ & \\
\hline
\end{tabular}

\section{RESULTS AND DISCUSSION}

X-Ray crystallographic analysis revealed the crystal structure of the title compound. And the structure is shown in Fig. 1. Selected bond distances and angles are listed in Table2. The single crystal structure of the title compound is built up by only the $\mathrm{C}_{22} \mathrm{H}_{28} \mathrm{~N}_{2} \mathrm{O}_{6}$ molecule. The title compound is a typical salen-type bisoxime derivative with normal geometric parameters. The molecule has crystallographic inversion symmetry. The molecule adopts an E configuration with respect to the azomethine $\mathrm{C}=\mathrm{N}$ bond and the two phenyl rings are paralle to each other.
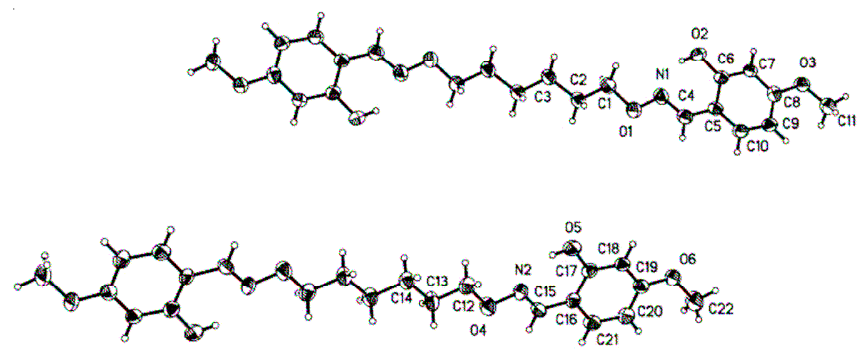

Fig. 1. Molecule structure of the title compound with atom numbering scheme. Displacement ellipsoids for non-H atoms are drawn at the $30 \%$ probability level

In the crystal structure, intramolecular hydrogen bonds $\mathrm{O} 2-\mathrm{H} 2 \cdots \mathrm{N} 1$ and $\mathrm{O} 5-\mathrm{H} 5 \cdots \mathrm{N} 2$ (Table-3) are observed between the hydroxyl groups and the oxime $\mathrm{N}$ atoms, the $\mathrm{O}-\mathrm{N}$ distances between the hydroxyl groups and the oxime $\mathrm{N}$ atoms are 2.658(3) $\AA$, indicating strong hydrogen bonding interactions $^{12-15}$. Moreover, each compound molecule links four other neighbouring molecules through two pairs of intermolecular

TABLE-2

SELECTED BOND DISTANCES $(\AA)$ AND ANGLES $\left({ }^{\circ}\right)$ FOR THE TITLE COMPOUND

\begin{tabular}{|c|c|c|c|c|c|}
\hline Bond & Lengths & Bond & Lengths & Bond & Lengths \\
\hline $\mathrm{N}(1)-\mathrm{C}(4)$ & $1.273(5)$ & $\mathrm{O}(6)-\mathrm{C}(22)$ & $1.405(5)$ & $\mathrm{C}(12)-\mathrm{C}(13)$ & $1.476(6)$ \\
\hline $\mathrm{N}(1)-\mathrm{O}(1)$ & $1.413(4)$ & $\mathrm{C}(1)-\mathrm{C}(2)$ & $1.504(6)$ & $\mathrm{C}(13)-\mathrm{C}(14)$ & $1.497(6)$ \\
\hline $\mathrm{N}(2)-\mathrm{C}(15)$ & $1.268(5)$ & $\mathrm{C}(2)-\mathrm{C}(3)$ & $1.511(6)$ & C(14)-C(14) & $1.524(8)$ \\
\hline $\mathrm{N}(2)-\mathrm{O}(4)$ & $1.401(5)$ & $\mathrm{C}(3)-\mathrm{C}(3)$ & $1.519(8)$ & $C(15)-C(16)$ & $1.445(6)$ \\
\hline $\mathrm{O}(1)-\mathrm{C}(1)$ & $1.428(5)$ & $C(4)-C(5)$ & $1.443(6)$ & $C(16)-C(21)$ & $1.392(5)$ \\
\hline $\mathrm{O}(2)-\mathrm{C}(6)$ & $1.355(5)$ & $C(5)-C(10)$ & $1.372(6)$ & $\mathrm{C}(16)-\mathrm{C}(17)$ & $1.429(6)$ \\
\hline $\mathrm{O}(3)-\mathrm{C}(8)$ & $1.375(5)$ & $C(5)-C(6)$ & $1.421(6)$ & $\mathrm{C}(17)-\mathrm{C}(18)$ & $1.361(6)$ \\
\hline $\mathrm{O}(3)-\mathrm{C}(11)$ & $1.411(5)$ & $C(6)-C(7)$ & $1.368(6)$ & C(18)-C(19) & $1.373(6)$ \\
\hline $\mathrm{O}(4)-\mathrm{C}(12)$ & $1.429(5)$ & $C(7)-C(8)$ & $1.387(6)$ & $\mathrm{C}(19)-\mathrm{C}(20)$ & $1.398(6)$ \\
\hline $\mathrm{O}(5)-\mathrm{C}(17)$ & $1.349(5)$ & $\mathrm{C}(8)-\mathrm{C}(9)$ & $1.386(6)$ & $\mathrm{C}(20)-\mathrm{C}(21)$ & $1.411(6)$ \\
\hline $\mathrm{O}(6)-\mathrm{C}(19)$ & $1.349(5)$ & $C(9)-C(10)$ & $1.358(6)$ & - & - \\
\hline Bond & Angles & Bond & Angles & Bond & Angles \\
\hline $\mathrm{C}(4)-\mathrm{N}(1)-\mathrm{O}(1)$ & $111.7(4)$ & $\mathrm{O}(2)-\mathrm{C}(6)-\mathrm{C}(5)$ & $121.8(5)$ & $\mathrm{C}(21)-\mathrm{C}(16)-\mathrm{C}(15)$ & $119.5(6)$ \\
\hline $\mathrm{C}(15)-\mathrm{N}(2)-\mathrm{O}(4)$ & $111.7(4)$ & $C(7)-C(6)-C(5)$ & $120.5(5)$ & $C(17)-C(16)-C(15)$ & $122.6(5)$ \\
\hline $\mathrm{N}(1)-\mathrm{O}(1)-\mathrm{C}(1)$ & $108.7(4)$ & $C(6)-C(7)-C(8)$ & $119.9(5)$ & $\mathrm{O}(5)-\mathrm{C}(17)-\mathrm{C}(18)$ & $119.4(5)$ \\
\hline $\mathrm{C}(8)-\mathrm{O}(3)-\mathrm{C}(11)$ & $118.2(4)$ & $C(6)-C(7)-C(8)$ & $119.5(2)$ & $\mathrm{O}(5)-\mathrm{C}(17)-\mathrm{C}(16)$ & $120.3(5)$ \\
\hline $\mathrm{N}(2)-\mathrm{O}(4)-\mathrm{C}(12)$ & $110.8(4)$ & $\mathrm{O}(3)-\mathrm{C}(8)-\mathrm{C}(9)$ & $124.2(5)$ & $\mathrm{C}(18)-\mathrm{C}(17)-\mathrm{C}(16)$ & $120.3(5)$ \\
\hline $\mathrm{C}(19)-\mathrm{O}(6)-\mathrm{C}(22)$ & $119.7(4)$ & $\mathrm{O}(3)-\mathrm{C}(8)-\mathrm{C}(7)$ & $114.9(6)$ & $\mathrm{O}(6)-\mathrm{C}(19)-\mathrm{C}(18)$ & $117.3(5)$ \\
\hline $\mathrm{O}(1)-\mathrm{C}(1)-\mathrm{C}(2)$ & $108.8(4)$ & $\mathrm{C}(9)-\mathrm{C}(8)-\mathrm{C}(7)$ & $120.9(5)$ & $\mathrm{O}(6)-\mathrm{C}(19)-\mathrm{C}(20)$ & $122.4(5)$ \\
\hline $\mathrm{C}(1)-\mathrm{C}(2)-\mathrm{C}(3)$ & $113.4(4)$ & $\mathrm{C}(10)-\mathrm{C}(9)-\mathrm{C}(8)$ & $117.7(5)$ & $\mathrm{C}(16)-\mathrm{C}(21)-\mathrm{C}(20)$ & 121.3(5) \\
\hline $\mathrm{C}(2)-\mathrm{C}(3)-\mathrm{C}(3)$ & $112.3(5)$ & $\mathrm{C}(9)-\mathrm{C}(10)-\mathrm{C}(5)$ & $124.5(5)$ & - & - \\
\hline $\mathrm{N}(1)-\mathrm{C}(4)-\mathrm{C}(5)$ & $122.7(5)$ & $\mathrm{O}(4)-\mathrm{C}(12)-\mathrm{C}(13)$ & $109.4(4)$ & - & - \\
\hline$C(10)-C(5)-C(6)$ & $116.5(5)$ & $\mathrm{C}(12)-\mathrm{C}(13)-\mathrm{C}(14)$ & 113.2(4) & - & - \\
\hline $\mathrm{C}(10)-\mathrm{C}(5)-\mathrm{C}(4)$ & $121.4(5)$ & $\mathrm{C}(13)-\mathrm{C}(14)-\mathrm{C}(14)$ & $114.4(5)$ & - & - \\
\hline $\mathrm{C}(6)-\mathrm{C}(5)-\mathrm{C}(4)$ & $122.0(5)$ & $\mathrm{N}(2)-\mathrm{C}(15)-\mathrm{C}(16)$ & $122.1(5)$ & - & - \\
\hline $\mathrm{O}(2)-\mathrm{C}(6)-\mathrm{C}(7)$ & $117.6(5)$ & $\mathrm{C}(21)-\mathrm{C}(16)-\mathrm{C}(17)$ & $117.8(5)$ & - & - \\
\hline
\end{tabular}


TABLE-3

HYDROGEN BONDS $\left[\AA{ }^{\circ}{ }^{\circ}\right]$ FOR THE TITLE COMPOUND

\begin{tabular}{|c|c|c|c|c|}
\hline $\mathrm{D}-\mathrm{H} \cdots \mathrm{A}$ & $\mathrm{d}(\mathrm{D}-\mathrm{H})$ & $\mathrm{d}(\mathrm{H} \cdots \mathrm{A})$ & $\angle \mathrm{DHA}$ & $d(D \cdots A)$ \\
\hline $\mathrm{O} 2-\mathrm{H} 2 \cdots \mathrm{N} 1$ & 0.82 & 1.94 & 146 & $2.658(3)$ \\
\hline $\mathrm{O} 5-\mathrm{H} 5 \cdots \mathrm{N} 2$ & 0.82 & 1.91 & 147 & $2.634(3)$ \\
\hline C11-H11C…O5 & 0.96 & 2.69 & 149 & $3.550(3)$ \\
\hline 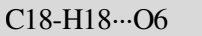 & 0.93 & 2.52 & 161 & $3.411(3)$ \\
\hline
\end{tabular}

C11-H11C ...O5 and C18-H18...O6 hydrogen-bonding interactions into an infinite wave-like 2D-layer supramolecular structure. The packing arrangement of the unit cell of the title compound is given in Fig. 2.

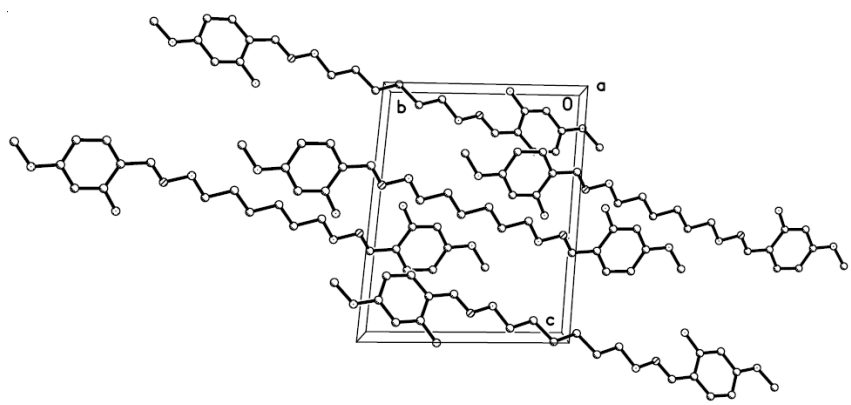

Fig. 2. Packing arrangement of the unit cell of the title compound. $\mathrm{H}$ atoms are omitted for clarity

\section{REFERENCES}

1. J.P. Costes, F. Dahan and A. Dupuis, Inorg. Chem., 39, 165 (2000).

2. W.K. Dong, L. Li, Y.X. Sun, J.F. Tong and J.C. Wu, Synth. React. Inorg. Met.-Org. Nano-Met. Chem., 41, 716 (2012).

3. W.K. Dong, J. Yao, Y.X. Sun, L. Wang, Y.J. Zhang and X.H. Gao, Synth. React. Inorg. Met.-Org. Nano-Met. Chem., 42, 408 (2012).

4. W.K. Dong, S.T. Zhang, Y.X. Sun, Y. Wang and L. Wang, Synth. React. Inorg. Met.-Org. Nano-Met. Chem., 41, 583 (2012).

5. W.K. Dong, Y.X. Sun, C.Y. Zhao, X.Y. Dong and L. Xu, Polyhedron, 29, 2087 (2010).

6. E.C. Niederhoffer, J.H. Timmons and A.E. Martell, Chem. Rev., 84, 137 (1984)

7. W.K. Dong and J.H. Feng, Acta Crystallogr., E62, o3577 (2006).

8. W.K. Dong, J.H. Feng and X.Q. Yang, Z. Kristallogr. NCS, 221, 447 (2006).

9. P.G. Lacroix, Eur. J. Inorg. Chem., 2, 339 (2001).

10. W.K. Dong, G. Wang, S.S. Gong, J.F. Tong, Y.X. Sun and X.H. Gao, Transition Met. Chem., 37, 271 (2012).

11. W.K. Dong, X.N. He, H.B. Yan, Z.W. Lv, X. Chen, C.Y. Zhao and X.L. Tang, Polyhedron, 28, 1419 (2009).

12. H.L. Wu, X.C. Huang, J.K. Yuan, F. Kou, F. Jia, B. Liu and Y. Bai, Z. Naturforsch., 66b, 1049 (2011).

13. W.K. Dong, G. Wang, S.S. Gong, J.F. Tong, Y.X. Sun and X.H. Gao, Transition Met. Chem., 37, 271 (2012).

14. W.K. Dong, Y.X. Sun, S.J. Xing, Y. Wang and X.H. Gao, Z. Naturforsch., 67b, 197 (2012).

15. W.K. Dong, L. Li, Y.X. Sun, S.T. Zhang, L. Wang, X.Y. Dong and X.H. Gao, J. Coord. Chem., 65, 2332 (2012). 